\title{
Atención Psicológica Postdesastres: Más que un "Guarde la Calma". Una Revisión de los Modelos de las Estrategias de Intervención.
}

\section{Post-Disaster Psychological Attention: More than a "Stay Calm". A Review of Intervention Strategics Models}

\author{
Claudia Pineda Marín* \\ Pontificia Universidad Javeriana \\ Wilson López-López ${ }^{* *}$ \\ Pontificia Universidad Javeriana
}

(Rec: 5 de agosto de 2010 / Acep: 21 de octubre de 2010)

\begin{abstract}
Resumen
El presente artículo expone algunos de los conceptos que sobre las estrategias de atención posterior a desastres que han sido publicadas en los últimos 5 años en PsicoRedalyc y APA PsyArticles. De la revisión se destaca el hecho de que en habla hispana las publicaciones son mínimas en contraste con las más de 100 catástrofes ocurridas entre 2001 y 2010. Se presentan también modelos sistematizados sobre la experiencia de catástrofes ocurridas alrededor del mundo y de las que se han generado diferentes guías para la asistencia de los profesionales en salud mental a las víctimas. Se distingue una tendencia en la aplicación de técnicas propias de las terapias cognitivo-conductuales y un interés en la prevención del Trastorno de Estrés Post Traumático.

Palabras clave: Intervención en crisis, catástrofes, desastres, modelos de intervención, atención psicológica, estrés post traumático, primeros auxilios psicológicos, terapia cognitivo-conductual,
\end{abstract}

\begin{abstract}
This article describes some of the concepts of care strategies after disasters that have been published over the past 5 years in PsicoRedalyc and APA PsyArticles. From the review highlights the fact that Hispanic publications are minimal in contrast to the more than 100 disasters occurred between 2001 and 2010. Systematized models are also presented on the experience of disasters occurring around the world and which have generated different guidelines for the care of mental health professionals to victims. It distinguishes a trend in the application of techniques of cognitive-behavioral therapy and an interest in the prevention of Post Traumatic Stress Disorder.

Key words: Crisis intervention, disasters, disaster, models of intervention, psychological care, post traumatic stress, psychological first aid, cognitive behavioral therapy,
\end{abstract}




\section{Introducción}

A lo largo de la historia el mundo ha experimentado la fuerza de la naturaleza y la magnitud de los desastres que causan los repentinos cambios ambientales no solamente afectan económica, social y físicamente un territorio sino a quienes se convierten en víctimas sobrevivientes de estos eventos inesperados. Un desastre es entendido como un evento o conjunto de eventos, causados por la naturaleza (huracanes, terremotos, inundaciones, etc.) o por actividades humanas (incendios, accidentes de transporte, etc.), durante el cual hay pérdidas humanas y materiales tales como muertos, heridos, destrucción de bienes, interrupción de procesos socioeconómicos y daños en la salud mental; además este tipo de emergencias tienen la particularidad de sobrepasar la capacidad de respuesta local y obligar a quienes la enfrentan a pedir ayuda a entidades externas (Figueroa, Marín y Gonzales, 2010).

Según el informe del Programa Panamericano de Defensa y Desarrollo de la Diversidad biológica, cultural y social, (s.f.), el cambio climático es y será uno de los mayores responsables de los desastres naturales, y en contraste con el informe de la Federación Internacional de Sociedades de la Cruz Roja y de la Media Luna Roja (s.f.), se prevé que los costos por desastres climáticos superarán diez veces la ayuda reservada para los países subdesarrollados, quienes son los que se verán enfrentados a las peores consecuencias. Solamente en lo corrido del año 2010 y en lo que respecta a América, la Cruz Roja reporta la probabilidad de que entre Junio y Noviembre de 2010, 8 a 14 de las 23 tormentas en Estados Unidos puedan convertirse en huracanes; sin olvidar eventos como el reciente terremoto en Chile ocurrido el 27 de Febrero pasado, que dejó más de 400 víctimas mortales y miles de damnificados y heridos; o el terremoto de 7,3 grados en la escala de Richter que sacudió a Haití el 12 de Enero de este mismo año, en el que se recuperaron 217.000 cuerpos y un número aproximado de 1.000.000 de damnificados. Al respecto de estas situaciones que parecen estar fuera de control, el Caribean Development Bank publica por primera vez en el 1998 el Natural Disaster Management, como un intento de mitigar los daños causados por los desastres naturales y a su vez una manera de prevenirlos; esto hace pensar que en efecto las cifras sobre el daño ambiental y sus consecuencias traducidas en catástrofes se han tornado uno de los temas de mayor interés para la comunidad científica en general, desde las agencias gubernamentales hasta los expertos en seguros tienen su atención fija en estos fenómenos (Caribean Development Bank, 2004). Así pues la producción de conocimiento psicológico acerca de la atención posterior al desastre no puede ser ajena al sufrimiento humano y fija su objetivo en la intervención en crisis producida por eventos circunstanciales y severos, como pueden serlo un terremoto, una erupción volcánica, una tormenta, etc.
Lo inmediatamente anterior haría pensar que desastres causados por el hombre, sean por acción directa o negligencia, no son catástrofes, pero eventos tales como el carro bomba accionado en Bogotá el 12 de Agosto de 2010 o la explosión de la mina de carbón en Amagá, Antioquia (Colombia), ocurrida a mediados de Junio de 2010, aunque son una verdadera tragedia, según el Centro de Colaboración de la Organización Mundial de la Salud para la Investigación de la Epidemiología de los Desastres (s.f.), no son catástrofes, pues para ser llamados así debe cumplir con 4 criterios, a saber, haber cobrado 10 o más vidas, haber afectado a 100 o más personas, haber obligado a declarar estado de emergencia, o haber obligado a solicitar apoyo internacional. Evidentemente los eventos catastróficos por lo inesperado y sorpresivo de su ocurrencia son sucesos que tienen la propiedad de desatar crisis y sufrimiento humano, es por eso que la psicología, en especial la clínica, ha dedicado gran parte de su trabajo a la atención de las víctimas de los desastres y en algunas ocasiones a la preparación de las personas en caso de que se vean expuestos a estas situaciones.

Aunque los desastres ocurren en todos los rincones del mundo, la producción escrita acerca de la atención psicológica se concentra -según las publicaciones de PsicoRedalyc y PsyArticles- en los países anglosajones; de los 35 artículos revisados y relacionados con la atención en desastres y las consecuencias psicológicas de las víctimas, solo 3 son producidos en Latinoamérica. El más antiguo de los artículos latinoamericanos fue producido en 2005 y el más reciente en 2010, sin embargo hay que resaltar que en 5 años solo estos 3 artículos están en las bases de datos antes mencionadas, que entran en fuerte contraste con las 112 emergencias reportadas por la Federación Internacional de Sociedades de la Cruz Roja y de la Media Luna Roja entre 2001 y 2010.

Lo anterior no supone que el quehacer psicológico no haya estado presente, sino que los aprendizajes y memorias de lo realizado en estos eventos no se ha sistematizado, escrito o publicado en revistas de alto impacto, aminorando las probabilidades de replicar experiencias y mejorar la eficacia de los tratamientos posteriores al desastre. En adelante, este escrito presentará algunos conceptos básicos que exponen los artículos revisados y los modelos que se proponen aplicar, sus convergencias y divergencias, y en general se propone sintetizar el aporte a la producción latinoamericana sobre atención psicológica post desastres.

\section{Modelos de las estrategias de intervención post desastres}

\section{Sobre cómo entender las crisis.}

Las crisis son definidas como "un estado temporal de trastorno y desorganización” (Slakeu, 2000, p. 16), en el 
que la situación desborda la capacidad de afrontamiento que tiene el sujeto y sus repertorios aprendidos no le sirven para solucionar el evento u obtener resultados positivos. Las crisis pueden ser desencadenadas por transiciones propias del ciclo vital o por eventos circunstanciales tales como desastres naturales o provocados por el hombre. Estos últimos son los que ocupan esta revisión y tienen características distintivas, como son el hecho de afectar a muchas personas al mismo tiempo, tener un carácter repentino, un impacto poderoso, percepción de incapacidad de control, pérdida de la confianza de las víctimas y persistencia de los efectos (Reyes y Elhai, 2004; Roberts, 2000).

Según Figueroa et al. (2010), aunque no necesariamente todas las víctimas de desastres sufrirán psicopatologías, la atención en primeros auxilios psicológicos y la intervención con terapias centradas en el trauma son una tarea indispensable para disminuir el impacto del desastre. Por su parte Pereira (2005), expone que algunas de las consecuencias psicológicas encontradas posterior al desastre aéreo ocurrido en 1996 en Sao Paulo, en la que fallecieron 99 personas, síntomas como disturbios en el sueño, irritabilidad, hostilidad en las relaciones con los otros y explosiones de rabia, dificultades en la concentración, hipervigilancia, desconfianza, sensación de inseguridad, respuestas exageradas a los estímulos del ambiente y en general varios síntomas que coinciden con los de los traumas. De igual manera, Slakeu (2000) afirma que los síntomas posteriores a un desastre son concomitantes con la ansiedad y la depresión.

Esto hace pesar que aunque la variedad en los eventos desencadenantes de crisis es múltiple, incluso dentro de los mismos desastres la crisis se puede desatar por la pérdida de vidas, las pérdidas materiales, las desapariciones, etc., y hay ciertas características similares en la vivencia de las crisis, ellas son: Desorden, Negación, Intrusión, Translaboración y Terminación. El desorden es entendido como el conjunto de conductas emitidas justo después del desastre; la negación, que puede funcionar como una manera de atenuar el impacto del desastre, consiste en un intento de omisión de la realidad latente por el desastre; la intrusión se define como un abarrotamiento de ideas y sentimientos de dolor, aparición de pesadillas y flashbacks, entre otros; en la translaboración las personas logran expresar los pensamientos, los sentimientos e imágenes que habían permanecido guardadas y que ahora empiezan a tener un nuevo significado; finalmente la terminación llamada también nuevo comienzo (Bridges, 1980/1982), consiste en un momento de reintegración u adaptación de la experiencia vivida a la historia de vida. Aunque no necesariamente todas las víctimas de un evento catastrófico viven todas estas fases de las crisis, los modelos de atención aquí citados sí se han creado a partir de estas categorías de transición; todas con el objetivo de responder tanto a las necesidades inmediatas como de procurar la salud mental de las personas (Slakeu, 2000).

\section{Modelos de intervención post desastres.}

Debido a que los efectos de los desastres son tan múltiples como sus causas, y estas pueden servir de instigador para mejorar o empeorar, en la producción escrita cuyo interés es el mejoramiento se indica que los esfuerzos en la formulación de modelos de intervención sobrepasan los primeros auxilios y se extienden en el tiempo y la profundidad. Pereira (2005) propone un modelo de intervención con 3 momentos, a saber: 1) restauración del dominio y funcionamiento cognitivo de las emociones; 2) Restauración del funcionamiento de las instituciones sociales y comunitarias, y 3) facilitar el reconocimiento cognitivo de lo que acontece. Rodríguez y Terry $(2003 ; 2005)$, en su experiencia sobre el sismo ocurrido en el eje cafetero colombiano, proponen: 1) Gestión del Riesgo que incluye atención en salud a las personas y atención en salud al medio ambiente; 2) Fase de sostenibilidad en salud física, social y mental; 3) Fase de estabilización en la que se decide cómo continuar con las acciones de atención y reconstrucción.

Otro de los modelos hallados en la literatura sobre la intervención posdesastres propone 5 niveles de atención, en los que se explicitan objetivos, beneficiarios, tareas específicas, ejecutores, lugar físico y criterios de derivación al siguiente nivel. Los niveles son: 1) Difusión, que cosiste en poner en conocimiento de la comunidad afectada la disposición del servicio de atención psicológica; 2) Apoyo social, cuyo objetivo es la identificación de las personas que están en riesgo de desarrollar alguna psicopatología y remitirlos a la unidad médica psiquiátrica; 3 ) Manejo médico general, en el que se estabilizan los síntomas psicológicos invalidantes, se realiza el diagnóstico psiquiátrico y se remite a psiquiatría si es pertinente; 4) Manejo psiquiátrico general, en este nivel se profundiza en la intervención psiquiátrica y se realiza a manos de expertos, las técnicas más utilizadas para este nivel son las cognitivo-conductuales o Eye movement desesitization and reprocessing (EMDR); y 5) Manejo psiquiátrico por expertos, este nivel incluye la administración de fármacos inhibidores del tipo mono-aminooxidasa, teniendo en cuenta que este es el último recurso para la atención y debe hacerse en centros especializados en Trastorno por Estrés Post Traumático (Figueroa et al., 2010).

A partir de una revisión publicada en 2004, Reyes et al., presentan 5 tipos diferentes de intervención para la atención en desastres. En primer lugar la Atención en Crisis centrada en mitigar el estrés emocional, vincular a los sobrevivientes con los recursos disponibles en la comunidad, y ayudar en la creación de un plan de recuperación a corto plazo en el que se aseguren las necesidades básicas para procurar un bienestar futuro. En segundo lugar la Psicoeducación, como herramienta de prevención para el actuar postdesastre, se propone preparar a las poblaciones en riesgo para afrontar los desastres de manera asertiva, clara y eficaz; esta preparación se dirige a líderes comunitarios y se difunde en todos 
los medios posibles. En tercer lugar los autores presentan el Debriefing Psicológico, cuya intención es que por medio de un interrogatorio dirigido por un profesional las personas expresen la manera como vivieron la experiencia del desastre y se realiza de manera individual o en grupo. El Defusing como cuarta estrategia, se propone -a manera de metáfora- desactivar el mecanismo que pueda activar "la bomba" de la psicopatología, facilitándole a la víctima del desastre un espacio para la expresión emocional. Finalmente los Primeros Auxilios Psicológicos, no necesariamente provistos por los psicólogos sino por cualquier persona entrenada, tienen la intención de proporcionar apoyo, evitar la muerte y direccionar a la persona hacia un servicio más profundo y especializado.

Después del Tsunami ocurrido en Tailandia, en Diciembre de 2004, investigadores alemanes propusieron un sistema de intervención por equipos; las tareas de los equipos de intervención de crisis se planearon así: Intervención en crisis para las víctimas y sus familiares, atención psicológica a los miembros de los grupos de rescate, atención para los grupos de identificación de las víctimas y también para el personal diplomático y el grupo de levantamiento; Recopilación de información para la planificación de nuevas medidas de intervención en crisis y el suministro de material informativo, y por último Atención a los familiares que viajan al país a buscar a familiares y amigos por su cuenta. Aunque este modelo de intervención probó ser muy eficaz en primera instancia, se recomendó que para posibles réplicas se hiciera un seguimiento de los casos tratados. En este modelo se resalta no solo la atención a las víctimas directas sino también la preocupación por el bienestar de quienes atienden en crisis y la intención de evaluar sistemáticamente un procedimiento de atención post-desastres (Bronisch, et al., 2006).

Por su parte Giannopoulou, Dikaiakou, \& Yule (2006) demostraron que la intervención grupal de tipo cognitivoconductual -dirigida a niños-, posterior al terremoto del 7 de septiembre de 1999 en Atenas, resultó ser un tratamiento eficaz para los niños afectados. Entre los efectos de la intervención se resaltan la reducción estadísticamente significativa del conjunto de síntomas del Trastorno por Estrés Post Traumático (intrusión, evitación, y excitación), así como en los síntomas depresivos. Aunque por la naturaleza del desastre y el componente ético que obliga a atender a todos quienes piden ayuda, no fue posible tener un grupo de control en la investigación, los autores reportan que no hubo cambios entre las medidas de referencia del inicio y del final de un periodo de espera en el grupo de tratamiento tardío.

El programa de tratamiento usado consistió en primer lugar en una sesión de introducción solo con los padres, cuyos objetivos eran por una parte normalizar las reacciones de sus hijos, informar a los padres sobre las estrategias de auto-ayuda, que les permitan ayudar a sus hijos y conocer qué tanto deberían involucrarse con la tarea de los niños.
Ya en la tarea con los niños se realizaron 7 sesiones; en la primera, se hicieron acuerdos para el tratamiento, se creó una definición del TEPT, y se ejemplificaron las posibles reacciones de los niños ante el desastre, esto con el fin de normalizar los comportamientos propios de la situación, y también hubo oportunidad de compartir la experiencia en grupo. En la sesión 2, y debido a los síntomas intrusivos presentados en la primera sesión, se trabajó la imaginería con el objetivo de generar una sensación de control sobre los flashbacks. En la tercera sesión el objetivo estaba fijado en los síntomas de excitación, para los cuales se entrenó a los niños en la identificación de sus reacciones y habilidades para relajarse a voluntad. En el cuarto encuentro se trataron las conductas evitativas, se trabajó con técnicas como el "termómetro del miedo", el establecimiento de jerarquías y técnicas de relajación, entre otras. En las dos sesiones siguientes ( 5 y 6 ), se realizó una exposición gradual mediante la imaginería, se utilizaron el dibujo, la comunicación verbal y la escritura como medios de expresión de la experiencia vivida. En la última sesión, la número 7, el objetivo se centró en resumir, reforzar y promover la generalización del uso de técnicas de manejo de la ansiedad, y la exposición, con énfasis en la prevención de recaídas, y terminó con un ejercicio de planificación para el futuro (Giannopoulou, et al., 2006).

Como se explicó anteriormente, las crisis también pueden ser desencadenadas por catástrofes causadas por acciones humanas directas, como en el caso del terrorismo. Sobre este tema las publicaciones evidencian un gran interés en la intervención, justo después del atentado del 11 de Septiembre de 2001 dirigido a los Estados Unidos. Sobre la intervención en estos casos Walser, Ruzek, Naugle, Padesky, Ronell, y Ruggiero (2004) proponen al igual que Giannopoulou et al. (2006) una intervención basada en los principios de la terapia cognitiva-conductual, que actúa en el corto y el mediano plazo. En el corto plazo, la intervención tiene dos fases, la primera es la Fase de Intervención en Emergencia, y en ella se parte de que las conductas emitidas inmediatamente después del evento de terror suelen ser desadaptadas, mas no síntomas de enfermedad mental, esto converge con el significado de la naturalización. En esta misma fase, se acompaña y guía a los afectados a afrontar con eficacia la situación, identificando las situaciones que le angustian y proponiéndole tareas concretas, alcanzables y realistas en el corto plazo que solucionen su dificultad; eso les permite a las personas recuperar el control de su situación. En esta fase también se les enseña a los individuos a manejar las emociones y sensaciones experimentadas al recordar el evento y se entrenan en habilidades para el manejo de la ansiedad, la respiración y la relajación.

En segundo lugar se habla de una Fase Aguda, cuyo objetivo es la prevención e intervención en los síntomas posteriores al trauma (incluyendo la prevención del TEPT), la mejoría en la motivación para la búsqueda de apoyo 
psicológico, la reducción de la activación psicológica (pues varios estudios han demostrado que el control temprano de la ansiedad puede detener el desarrollo del TEPT) (La Greca, Silverman, Vernberg y Prinstein, 1996), el aumento de la capacidad de afrontamiento (búsqueda de apoyo, dar apoyo, resolución de problemas y comunicación en general) por medio de la especificación cuidadosa de los comportamientos deseados, el uso de auto-monitoreo y el refuerzo social frente a los esfuerzos, de igual manera el auto -control permite a las personas reconocer sus propios esfuerzos, reflexionar sobre la situación y sus posibles acciones. En esta fase también se procura la reducción de conductas de enfrentamiento que pueden no ser adaptativas en el mediano plazo o contraproducentes en la superación de la crisis, tales como el consumo del alcohol o drogas; estas actividades pueden dificultar los roles sociales y las habilidades para la recuperación (North, Tivis, McMillen, Pfefferbaum, Spitznagel, Cox, Nixon, Bunch, y Smith, 2002; Heather,1995). También se trabaja la reestructuración de las creencias negativas relacionadas con el trauma, pues es posible que los juicios sobre el valor de la experiencia y la incidencia en el futuro impidan la recuperación y prolonguen el sufrimiento. Por último esta fase se propone la facilitación del "procesamiento de la emoción", es decir la exploración y exposición sistemática al trauma (Walser et al., 2004).

En el largo plazo Walser et al. (2004) formulan una Intervención Secundaria, para el tratamiento de los síntomas, siendo la terapia cognitivo-conductual la que ha demostrado mayor eficacia en el tratamiento de TEPT, desde la detección hasta la aplicación de métodos propios para el TEPT causado por acciones terroristas, pasando por la exposición básica, la exposición prolongada o gradual y la desensibilización sistemática. Para la intervención en TEPT causado por acciones terroristas Ehlers y Clark (2000), presentan un modelo de intervención que demostró ser eficaz en el tratamiento de quienes fueron heridos, testigos o personal de emergencia del hospital, afectados por un atentado terrorista perpetrado en Omagh, Irlanda del Norte en 1998, en el que 29 personas murieron y más de 370 resultaron heridas. En síntesis los autores aplicaron un modelo que consistía en 3 etapas. 1. Identificación y confrontación de las creencias negativas sobre los efectos a largo plazo del trauma y los síntomas; 2. Organización y recolección de la memoria sobre el trauma con el fin de integrarla en la vida de cada una de las personas expuestas al evento, y 3. Superación de la conducta evitativa, de seguridad, y las reflexiones que impiden la elaboración de la memoria, exacerban los síntomas o interfieren con la resignificación de las creencias. Esto también se puede lograr con el reintegro de las personas a las actividades que realizaban antes del acto terrorista. Los autores proponen una identificación de las emociones seguida de una reestructuración cognitiva de los significados y la reinserción de estos significados reestructurados en el recuerdo del evento traumático, esto puede mejorar de manera significativa la eficacia del tratamiento del TEPT. De igual manera el impacto emocional que ocasionan las crisis no solo puede desatar un TEPT, sino en muchos casos podrían tener un carácter psicosomático, siendo la razón por la cual aparecen cefaleas, dolores abdominales, entre otras molestias, que son la respuesta a un mayor nivel de sensibilidad fisiológica y una acrecentada percepción de las señales corporales. En un estudio realizado por Barra-Almagia (2008) los síntomas más frecuentes reportados por los adolescentes expuestos a situaciones de estrés fueron los resfríos, el dolor de cabeza y el dolor de estómago.

De esta manera se recogen algunos de los aportes que los expertos en psicología clínica sistematizan sobre la experiencia y la intervención posterior a los desastres, que hoy por hoy son más frecuentes, dadas las condiciones ambientales que los potencializan y la evidente omisión de programas de prevención del TPEP en comunidades de riesgo.

\section{Conclusiones}

Weiji es el término chino de crisis, y está compuesto por dos caracteres cuyo significado es peligro y oportunidad (Wilhem, 1967, citado en Slaikeu, 2000). Peligro, por las consecuencias de las pérdidas, y oportunidad, porque de ellas puede emerger el desarrollo. Si bien es cierto que las catástrofes son eventos traumáticos, causantes de fuertes lesiones en la economía, en la sociedad y en la salud mental, también lo es que desata crisis que pueden ser evento para el mejoramiento. En el caso de los modelos de intervención psicológica posterior a los desastres, ha sido una oportunidad para evolucionar y hacer modelos más eficaces en el tratamiento inmediato y la prevención de psicopatologías que se presentan en un lapso de tiempo más largo a la ocurrencia del evento traumático.

Esta revisión permite ver algunas convergencias entre los modelos, tanto en la manera de comprender los desastres como en sus constructos teóricos. En el primer caso, los modelos aquí presentados coinciden en la importancia de la atención psicológica temprana para restablecer el equilibrio emocional, utilizando técnicas que en su mayoría pertenecen al enfoque cognitivo-conductual (desensibilizacion sistemática, exposición gradual, entrenamiento en relajación, ejercicios de respiración, etc.), así mismo se resalta la importancia de la difusión de información respecto a la disponibilidad de un servicio de atención psicológica, con capacidad de apoyar el proceso de readaptación de niños, jóvenes y adultos. La intervención es fundamental en la prevención del desarrollo del TEPT, pues aunque no se presente inmediatamente, existe la probabilidad de que la catástrofe sirva como desencadenante a largo plazo de 
la psicopatología. De igual manera a partir del desastre las personas pueden incrementar el consumo de sustancias que tienen como función inmediata el control emocional, pero a la vez pueden ser una dificultad para el afrontamiento y la planeación del futuro (North, 2002; Heather,1995).

Estos dos últimos factores (el afrontamiento y la planeación del futuro) parecen ser los mejores indicadores de que la crisis podrá llegar a su fin y dar lugar a un "nuevo comienzo", y ese es uno de los principales objetivos de los modelos propuestos. Aun cuando algunos modelos no plantean el seguimiento a largo plazo del estado de los consultantes, se asume que con el control de los síntomas se inhibirá la aparición de la psicopatología y con ello se dará lugar a la restitución de la vida antes de la catástrofe. Probablemente haya daños irreparables después de los desastres, tales como pérdidas de familiares, amigos, etc., pero al igual que en las crisis generadas por las transiciones propias del ciclo vital, es posible la superación y el mejoramiento. En este mismo sentido se destaca el hecho de que las redes comunitarias de apoyo y el compromiso social por el progreso, tienen un nivel importante de incidencia en la superación de la crisis (Rodríguez y Terry 2003, 2005; Walser, Ruzek, Naugle, Padesky, Ronell, y Ruggiero,2004; Giannopoulou, Dikaiakou, y Yule, 2006)

Uno de los aspectos más importantes que dilucida esta revisión, es el hecho de que no existe o no es muy popular la educación en primeros auxilios psicológicos, como si lo son los primeros auxilios médicos. Esto indica por una parte que sí los profesionales a cargo de la salud mental y el sufrimiento humano no están presentes para atender a los afectados por los desastres, la probabilidad de que las conductas disfuncionales aparezcan es alta. Como es sabido, la ejecución de primeros auxilios no requiere profesionales, sino personas de comunidades entrenadas con instrucciones y protocolos de acción probadas con eficiencia, así que es tarea de los expertos preparar a las comunidades para una eventual catástrofe que requiera intervención psicológica, de esta manera no solo se haría posible una mayor cobertura en el servicio sino una mejor capacidad de afrontameinto.

En lo que a sistematización de experiencias se refiere, es claro que por la naturaleza de los desastres, la investigación en ese momento tenga limitaciones que impidan el control de los estándares de confiabilidad y validez de los métodos aplicados, no obstante los aportes y las memorias publicadas constituyen un importante peldaño en el avance y la creación de nuevas estrategias de intervención psicológica.

Para terminar hacemos énfasis en que las últimas catástrofes de que han sido testigos los latinoamericanos, y en las que además de cientos de víctimas mortales ha habido millares de damnificados, quienes vivieron muchas transiciones a la vez (duelos, pérdidas materiales, daño social e incluso sus propias transiciones vitales) demandan a la psicología una actuación responsable en la recuperación integral de la calidad de vida de nuestros habitantes.

\section{Referencias}

Barra-Almagia, E. (2008). Influencia del estrés y el ánimo depresivo sobre la salud adolescente: análisis concurrente y prospectivo. Universitas Psychologica, 8, 175-182.

Bridges, W. (1980/1982). Transiciones. El por qué de los cambios que vivimos. México: Fondo educativo interamericano.

Bronisch, T., Maragkos, M., Freyer, C., Müller-Cyran, A., Butollo, W., Weimbs, R., y Platiel, P. (2006). Crisis Intervention after the Tsunami in Phuket and Khao Lak. Crisis, 27, 42-47.

Caribean Development Bank (2004). Natural Disaster Management. Recuperado en Julio 30, 2010, disponible en http://www.caribank. org/titanweb/cdb/webcms.nsf/AllDoc/9FB25BD231DCD78704257 3CD0062F282/\$File/NatDisMngtStrat\&OpGdlnesPaper\%5B1\%5D. pdf?OpenElement

Centro de Colaboración de la Organización Mundial de la Salud para la Investigación de la Epidemiología de los Desastres (s.f.). Desastre Natural. Recuperado en Julio 30, 2010 disponible en http://www. worldlingo.com/ma/enwiki/es/Centre_for_Research_on_the_Epidemiology_of_Disasters

Ehlers, A., y Clark, D.M. (2000). A cognitive model of posttraumatic stress disorder. Behaviour Research and Therapy, 38, 319-345.

Federación Internacional de Sociedades de la Cruz Roja y de la Media Luna Roja. (s.f.) Emergencias. Recuperado en Julio 30, 2010 disponible en http://www.cruzroja.org/emergencias/

Figueroa, R., Marín, H., y Gonzales, M. (2010). Apoyo Psicológico en desastres: Propuesta de un modelo de atención basado en revisiones sistemáticas y metanálisis. Revista Medica de Chile, 138, 143-151.

Heather, N.(1995). Brief intervention strategies. In: R.M. Hester, W.R. Miller (eds), Handbook of Alcoholism Treatment Approaches: Effective Alternatives (pp 105-122). New York: Pergamon Press.

Giannopoulou, I., Dikaiakou,A., \& Yule, W. (2006). Cognitivebehavioural group intervention for PTSD symptoms in children following the Athens 1999 earthquake: A pilot study. Clinical Child Psychology and Psychiatry, 11, 543-553.

La Greca, A., Silverman, W., Vernberg E., y Prinstein, M. (1996). Symptoms of posttraumatic Stress in Children After Hurricane Andrew: A Prospective Study. Journal of Consulting and Clinical Psychology, 4. 712-723.

North, C.S., Tivis, L., McMillen, J.C., Pfefferbaum, B., Spitznagel, E.L., Cox, J., Nixon, S., Bunch, K.P., y Smith, E.M. (2002) Psychiatric disorders in rescue workers of the Oklahoma City bombing. American Journal of Psychiatry, 157, 857-859.

Pereira, M.H. (2005). Atendimento psicológico para emergencias em aviação; a teoría revista na práctica. Estudos de Psicología, 10, 177-180.

Programa Panamericano de Defensa y Desarrollo de la Diversidad biológica, cultural y social. (s.f.) Desastres Naturales en el planeta. Recuperado en Julio 30, 2010 disponible en http://www.prodiversitas. bioetica.org/des37.htm

Reyes, G., y Elhai, D. (2004). Psychosocial interventions in the early phases of disasters. Psychotherapy: Theory, Research, Practice, Training, 41, 399-411.

Rodriguez, A., y Terry, B. (2003). Terremoto en el eje cafetero colombiano: Lecciones aprendidas. Revista Española de Nutrición Comunitaria, 9, 182-4

Rodriguez, A., y Terry, B. (2005). Estrategia integral de atención en la posemergencia ante el sismo del eje cafetero colombiano [Versión electrónica], Revista Cubana Higiene y Epidemiología. 43 (2). Recuperado el 2 de Julio de 2010, de http://bvs.sld.cu/revistas/hie/vol43_2_05/ hie03205.pdf

Roberts, A. R. (2000). An overview of crisis theory and crisis intervention. En R. Roberts (ed.), Crisis intervention handbook: Assessment, treatment, and research (pp.3-30). New York: oxford university press.

Slaikeu, K. (2000). Intervención en Crisis. México: Manual Moderno.

Walser, R., Ruzek, J., Naugle, A., Padesky, C., Ronell, D., y Ruggiero, K. (2004). Disaster and Terrorism: Cognitive-Behavioral Interventions. Prehospital and Disaster Medicine, 19, 54-63. 